\title{
Review Article \\ Prostate Cancer: Epigenetic Alterations, Risk Factors, and Therapy
}

\author{
Mankgopo M. Kgatle, ${ }^{1}$ Asgar A. Kalla, ${ }^{2}$ Muhammed M. Islam, ${ }^{3}$ \\ Mike Sathekge, ${ }^{4}$ and Razia Moorad ${ }^{5}$ \\ ${ }^{1}$ Division of Hepatology and Liver Research, Department of Medicine, Faculty of Health Sciences, University of Cape Town and \\ Groote Schuur Hospital, Observatory, Western Cape 7925, South Africa \\ ${ }^{2}$ Division of Rheumatology, Department of Medicine, Faculty of Health Sciences, University of Cape Town and Groote Schuur Hospital, \\ Observatory, Western Cape 7925, South Africa \\ ${ }^{3}$ Department of Integrative Biomedical Sciences, Institute of Infectious Disease and Molecular Medicine, Faculty of Health Sciences, \\ University of Cape Town, Observatory, Western Cape 7925, South Africa \\ ${ }^{4}$ Department of Nuclear Medicine, University of Pretoria and Steve Biko Academic Hospital, Private Bag X169, Pretoria, \\ Gauteng 0001, South Africa \\ ${ }^{5}$ Department of Surgery, Faculty of Health Science, University of Cape Town and Groote Schuur Hospital, Observatory, \\ Western Cape 7925, South Africa
}

Correspondence should be addressed to Mankgopo M. Kgatle; mankgopo.kgatle@gmail.com

Received 21 June 2016; Accepted 4 October 2016

Academic Editor: Cristina Magi-Galluzzi

Copyright (C) 2016 Mankgopo M. Kgatle et al. This is an open access article distributed under the Creative Commons Attribution License, which permits unrestricted use, distribution, and reproduction in any medium, provided the original work is properly cited.

Prostate cancer $(\mathrm{PCa})$ is the most prevalent urological cancer that affects aging men in South Africa, and mechanisms underlying prostate tumorigenesis remain elusive. Research advancements in the field of $\mathrm{PCa}$ and epigenetics have allowed for the identification of specific alterations that occur beyond genetics but are still critically important in the pathogenesis of tumorigenesis. Anomalous epigenetic changes associated with PCa include histone modifications, DNA methylation, and noncoding miRNA. These mechanisms regulate and silence hundreds of target genes including some which are key components of cellular signalling pathways that, when perturbed, promote tumorigenesis. Elucidation of mechanisms underlying epigenetic alterations and the manner in which these mechanisms interact in regulating gene transcription in PCa are an unmet necessity that may lead to novel chemotherapeutic approaches. This will, therefore, aid in developing combination therapies that will target multiple epigenetic pathways, which can be used in conjunction with the current conventional PCa treatment.

\section{Introduction}

According to the World Cancer Research Fund's list, South Africa is ranked 50th among countries with the highest cancer prevalence [1]. Recent article published in Lancet projected cancer to become a leading cause of deaths in South Africa, with an increase from 77400 cases in 2008 to 112921 new cases in 2030 [2]. Prostate cancer (PCa) is the most prevalent urological cancer and one of the four top cancers including Kaposi sarcoma and colorectal and lung cancers, which affect aging men in South Africa [2]. It is estimated that 1 in 8 South African aging men will develop PCa at some point in their lifetime. The most common type of PCa is acinar adenocarcinoma, representing $90 \%$ of the PCa cases [3].

Prostate is a walnut sized organ located just between the bladder and penis, and it slowly grows larger to an average weight of 40 grams in aging men. A prostate gland surrounds the urethra that empties urine from the bladder and also secretes prostate fluid that protects sperm. These physiological functions may be compromised during various prostate diseases including prostatitis, benign prostatic hyperplasia or hypertrophy, and cancer. PCa begins when aberrant semensecreting prostate gland cells develop and proliferate uncontrollably. If left untreated, PCa may metastasise to other 
parts of the body, particularly to the lymph nodes and bones. Although most patients may remain asymptomatic in the early stages, advanced PCa may be accompanied by a variety of urinary symptoms including troublesome nocturia, dysuria, hematuria, hematospermia, pain and swelling in the legs and pelvic area, loss of bladder, and erection control. On the other hand, benign prostatic hyperplasia or hypertrophy may show similar symptoms but is rarely life-threatening. In this case, the use of total plasma prostate specific antigen (tPSA) level becomes important. According to ASC, a tPSA level of more than the cut-off value of $4 \mathrm{ng} / \mathrm{mL}$ may indicate the occurrence of PCa [4]. To overrule the possibility of an elevated tPSA level in a subset in nonmalignant conditions, transrectal biopsy guided by ultrasound can be used to validate the diagnosis $[4,5]$.

Owing to its slow growth, PCa may take up to 10 years to progress from precursor prostatic intraepithelial neoplasia (PIN) to an invasive carcinoma. Histologically, PIN can be classified into low and high grade, which are generally characterised by various molecular or cellular architecture [6]. The Gleason grading system developed by Dr. Donald F. Gleason between 1966 and 1974 and recently reviewed and improved by the 2014 International Society of Urological Pathology remains one of the most powerful predictors for the prognostic outcome of PCa. An alternative to the current Gleason score was recently proposed with the potential to lessen the overtreatment of low-grade PCa detected by PSA screening. The new simplified PCa grading incorporates five grades, and these include grade group 1 (Gleason score $\leq 6$ ), grade group 2 (Gleason score $3+4=7$ ), grade group 3 (Gleason score $4+$ $3=7$ ), grade group 4 (Gleason score 8 ), and finally grade group 5 (Gleason score 9-10) [7].

$\mathrm{PCa}$ is a heterogeneous disease, and its occurrence differs extremely from patient to patient even within the same tumour. The great disparity in the PCa architecture and incidence rates may be attributable to genomic instabilities and alterations associated with various PCa risk factors. Therefore, this review aims to provide a comprehensive understanding of the prostate carcinogenesis-related epigenetic signatures and their mediators. In addition, the associated PCa risk factors and recent treatment options are discussed.

\section{Current Prostate Cancer Treatment}

Depending on the severity of the disease, current therapies for PCa may include watchful waiting, hormone therapy, therapeutic vaccines, bone-directed treatment, cryotherapy, radiation therapy, and surgery. Although these treatment options may improve the quality of life of patients by significantly delaying or inhibiting the progression of the disease, chemotherapeutic resistance commonly develops often resulting in death $[8,9]$. In addition, an estimated $30 \%$ of patients relapse following the initial treatment. The 5-year survival rate for the localised and regional PCa is nearly $100 \%$; however, this percentage drops to $28 \%$ in cases where cancer has metastasised to distant organs $[9,10]$.

Two recently approved hormone therapy agents, the AR antagonist enzalutamide and the CYP17Al-inhibitor abiraterone, have proven to be well tolerated and effective

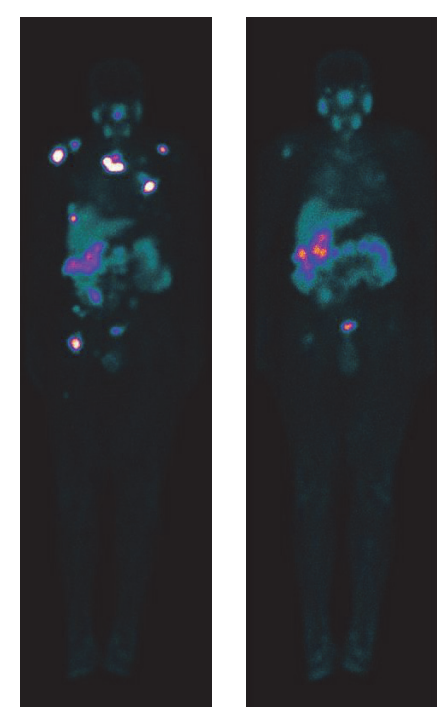

Figure 1: Anterior whole body ${ }^{177}$ Lu-PSMA scan of a 72 -yearold patient with metastasised castration-resistant prostate cancer. This demonstrates normal biodistribution in the nasal region, salivary glands, liver, and spleen gastrointestinal and urinary system, with multiple PSMA-avid metastases in the prostate bed, clavicle, sternum, clavicle, vertebrae, iliac bone, and femora. An excellent response to therapy was observed after 3 cycles of ${ }^{177}$ Lu-PSMA-RLT with decrease in serum PSA level (from 63 to $4.25 \mathrm{ng} / \mathrm{mL}$ ).

in many metastasised castration-resistant PCa patients [11]. Nonetheless, these therapy agents remain noncurative, suggesting an urgent need of novel systemic treatment that can improve the overall survival of PCa patients. This encouraged the development of novel therapeutic approaches such as the prostate specific membrane antigen-targeted radioligand therapy (PSMA-RLT) [12]. PSMA-RLT allows the delivery of high dose of therapeutic radiation to cancer cells, while lessening the exposure of normal cells. PSMA, also known as folate hydrolase I or glutamate carboxypeptidase II, is a cell surface protein that is significantly overexpressed in refractory or metastasised castration-resistant PCa cells. The PSMA-RLT involves binding of a ligand PSMA to a radioactive isotope $\mathrm{Lu}-177$, and this facilitates the detection of metastatic sites for treatment as illustrated in Figure 1. The widely used radiopharmaceutical is ${ }^{177} \mathrm{Lu}$-PSMA. The radionuclide is transported directly to the tumour cells and radioactive decay of ${ }^{177} \mathrm{Lu}$ emits beta particles, which are absorbed by the disease targeted sites thereby damaging the surrounding PCa cells $[13,14]$. PSMA-RLT with ${ }^{177} \mathrm{Lu}-$ PSMA holds a great promise to be a magic bullet for patients with metastasised castration-resistant $\mathrm{PCa}$ with appropriate selection and follow-up by ${ }^{68} \mathrm{Ga}$-PSMA PET/CT as it follows a theranostic approach, which is the basis for successful radionuclide therapy [14-16].

Radiomics is a high throughput approach used to extract a large number of qualitative and quantitative features from medical images, which could improve tumour phenotype characterisation and treatment outcome prediction [17]. Positive ${ }^{68} \mathrm{Ga}$-prostate-specific membrane antigen for diagnostics 
with positron emission tomography/computed tomography (PET/CT) has the ability to capture intratumoural heterogeneity in a noninvasive way as well as to assess the response to PSMA-RLT. This imaging has brought radiomics closer to reality when it significantly reduced false-negative biopsies by guiding the needle to the highest Gleason score lesion in a comparative trial of biopsied PCa patients [18]. ${ }^{68} \mathrm{Ga}-$ PSMA PET/CT imaging and the use of PSMA-RLT with ${ }^{177}$ Lu-PSMA may provide better risk stratification of PCa patients, if the findings are complemented with the knowledge of somatic mutations and epimutations. Recent data on a mouse model have shown that combining epigenetic drugs with immunomodulatory antibodies that target cytotoxic T-lymphocyte antigen 4 (CTLA-4) and programmed cell death 1 (PD-1) improves antitumor activity compared to the case when they were used individually [19]. For instance, syngeneic mammary and colorectal carcinoma models demonstrated that combination of 5-azacytidine (DNA demethylating agent) plus entinostat (histone deacetylase inhibitor) and CTL-4 plus CD-1 significantly improved therapeutic outcomes. The same study have also shown that anti-CD40 plus anti-CD137 immunotherapy combined with panobinostat (another histone deacetylase inhibitor) delayed tumour growth in syngeneic mammary (i.e., 4T1.2), colon (i.e., CT26), and prostate carcinoma mouse models [20].

\section{Epigenetic Signatures and Mediators in Prostate Cancer}

The epigenetic regulation of gene expression has been substantially studied for over 19 years now and is embraced as the well-known hallmark of tumorigenesis. Perturbed key multiple and important cellular processes that are normally suppressed or activated to inhibit malignant transformation is the common phenotype of aberrant gene expression. Cancerrelated epigenetics, also known as epimutations, involves anomalous epigenetic alterations of gene transcription in cancer cells that are not attributable to any change in the nucleotide sequence [21,22]. Advanced research in this rapidly growing and evolving landscape of cancer epigenetics has revealed a large amount of data showing global and specific epigenetic alterations, which are characterised by suppressed cell apoptosis, sustained cell proliferation, and invasion. These cellular manifestations are associated with hijacked or disrupted physiological pathways mediated by inactivating mutations and silencing of the transcriptional activities of cell-cycle activators [23, 24]. Several lines of evidence show that histone modifications, DNA methylation, and noncoding microRNA (miRNA/miR) are the most common epigenetic signatures responsible for the aberrant suppression of gene transcription observed in many malignancies. PCa is also influenced by a combination of genetic and/or epigenetic alterations that are strongly linked to perturbed cellular processes and tumour initiation. However, how these aberrant epigenetic signatures are established still remains poorly understood.

\section{DNA Methylation}

DNA methylation can be categorised as hypermethylation (increased methylation) and hypomethylation (decreased methylation). It involves chemical attachment of a methyl group to the carbon 5 on the genome, thereby leading to an alteration in gene transcription and normal function [21]. This phenomenon is established and maintained by various active DNA methyltransferase (DNMTs) enzymes including DNMT1, DNMT3A, and DNMT3B [21, 22, 25]. Hypermethylation is one of the best dissected epigenetic alterations in PCa and involves well-known genes that are important in DNA damage repair (GSTP1 and MGMT), apoptosis (DAPK1; RASSF1), hormonal response (androgen receptor- $A R$, estrogen receptor-ER, and $R A R \beta$ ), inflammatory responses (prostaglandin endoperoxide synthase 2-PTGS2), cell-cycle control (CDKN2A), and metastases (Cadherins, CD44, and tissue inhibitor metallopeptidase (TIMP)) [26]. Like in other malignancies, silence in gene expression is the most common trait associated with promoter hypermethylation in PCa. This is often highly detected in advanced pathologic grades or clinical stages and usually accompanied by cancer development, invasion, and metastasis. More recently, hypermethylation of genes such as Ephrin-A5 (EphA5) and transmembrane protease serine 2 (TMPRSS2) has been revealed in PCa. Aberrant hypermethylation of the Eph5A CpG promoter regions was observed in advanced $\mathrm{PCa}$ patients with a Gleason score of 8 . Eph $5 A$ is one of the Eph family of receptors of tyrosine kinases that are implicated in several human malignancies including PCa and hepatocellular carcinoma [27, 28]. TMPRSS2, an AR signalling downstream gene, is a type II transmembrane protease abundantly expressed in the prostate gland that has been shown to induce tumorigenesis when altered. DNMT1-induced hypermethylation at the promoter region of TMPRSS 2 was observed in PCa cells. This was accompanied by a suppressed expression of $A R$ and TMPRSS2, which was restored through treatment with demethylating agent 5 -Aza- $2^{\prime}$-deoxycytidine [28]. Overexpression of $E R \alpha$ is directly proportional to $\mathrm{PCa}$ disease progression and promotes oncogenic events such as fusion of TMPRSS2 and estrogen-regulated gene (ERG) located on chromosome 21. It was discovered that TMPRSS2 is fused to either ERG or ETS variant 1 (ETV1) and this frequent event contributes to PCa tumour progression [2931]. Global DNA hypomethylation was hypothesised to occur later in PCa than CpG island hypermethylation and therefore is more likely to contribute to PCa metastasis than initiation and progression. Genes that are hypomethylated in PCa cells are limited, and these include LINE1, X-inactive specific transcript, plasminogen activator urokinase, and heparanase [32].

Androgen receptors (ARs) play a fundamental role by interacting with testosterone or dihydrotestosterone, which are crucial for the development of the male reproductive system during embryogenesis and sexual development at puberty. In PCa, aberrant hypermethylation of the AR correlates with suppressed gene transcription and increased PCa cell growth and proliferation [33]. A significant difference in methylation profiles that affect genes including caspase 8, 
CD14, multiple drug resistant 1, and glypican 3 was observed between the AR-resistant and AR-negative cancer cells [34]. Matthew Freedman and his colleagues from Dana-Farber Cancer Institute and Harvard Medical School have recently shown in the human PCa tissues that the AR cistrome undergoes extensive reprogramming, which is an erasure and remodelling of DNA methylation [35]. This phenomenon was accompanied by localisation of forkhead Box A1 (FOXA1) and homeobox B13 (HOXB13) at the programmed AR binding sites, suggesting the implication of transcription factors in establishing aberrant epigenetic reprogramming [35].

The transcription factor E twenty-six-related gene (ERG) mediates epigenetic alterations in the AR cistrome and prime prostate tumorigenesis in a mouse model [36]. Ten-eleven translocation methylcytosine dioxygenase (Tet) proteins 1, 2 , and 3 were demonstrated to preserve the unmethylated CpG islands by converting 5-methylcytosine to hydroxymethylcytosine via oxidation induced through interaction and catalytic activities of iron and alpha-ketoglutarate. In PCa, androgen hormone induced global hydroxymethylation silences Tet 2 expression, leading to poor prognosis. This was detected largely among global FOXA1-binding sites and inhibited FOXA1 binding at specific loci, suggesting that alterations in Tet 2-mediated pathway may have a significant implication for treating advanced PCa [37].

\section{Interaction among Epigenetic Mechanisms in Prostate Cancer}

Histones commonly undergo extensive covalent modifications that alter chromatin structure or function, and these include methylation, acetylation, ubiquitylation, and sumoylation. Histone methylation/demethylation and acetylation/deacetylation are extensively studied [38, 39]. Histone acetylation (or deacetylation) involves the addition (or removal) of an acetyl group on lysine $(\mathrm{K})$ residues within the N-terminal tail protruding from the histone core of the nucleosome and is generally linked to gene activation. When methyl groups are added to (or removed from) histone protein amino acids and result in the transcriptional activation or silence, the process is known as histone methylation (or demethylation) [22]. Lysines can be mono-, di-, or trimethylated, and this can be associated with either silence or active in-gene transcription. For instance, methylation on $\mathrm{H} 3 \mathrm{~K} 4$ and $\mathrm{H} 3 \mathrm{~K} 36$ is associated with active gene transcription, whereas mono-, di-, and trimethylation on $\mathrm{H} 3 \mathrm{~K} 9, \mathrm{H} 3 \mathrm{~K} 27$, and H4K20 mark transcriptionally silent chromatin. Histone acetylation/deacetylation and methylation/demethylation are driven by histone acetyltransferases (HATs)/deacetylases (HDACs) and methyltransferases (HMTs)/demethylases [22, 25].

The polycomb group of proteins including polycomb repressive complex $1(\mathrm{PRC} 1)$ and $\mathrm{PRC} 2$ are key transcriptional repressor complexes that induce histone methylation to repress Hox genes involved in early development and stem cell differentiation. The main complex, PRC2, is composed of four core subunits: embryonic ectoderm development, suppressor of zeste 12, retinoblastoma binding protein 4, and enhancer of zeste 1 (EZH1) or EZH2 [40, 41]. PRC2 silences gene expression through trimethylation of $\mathrm{H} 3 \mathrm{~K} 27$, which is catalysed by its enzymatic units EZH1 and EZH2 $[42,43]$. Disrupted activity of EZH2 through aberrant histone methylation promotes transcriptional instability that favours tumorigenesis and drug resistance. In castration-resistant $\mathrm{PCa}$, overexpression of $\mathrm{EZH} 2$ via $\mathrm{H} 3 \mathrm{~K} 27 \mathrm{me} 3$ is associated with poor clinical outcome, prognosis, and metastasis [44]. The role of EZH2 in tumorigenesis was strengthened by its knockdown in both castrated xenograft mouse model and human PCa cells that led to androgen-independence growth arrest, significant reduction of tumour growth, and metastasis. The activity of EZH2 can also be regulated by other nonPRC2 independent pathways such as phosphatidylinositol3-kinases (PI3K)/AKT signalling pathways. Dysregulation in PI3K/AKT signalling pathway is associated with perturbed cellular processes including apoptosis, cell growth, and survival, which in turn leads to tumour progression. $\mathrm{PI} 3 \mathrm{~K} / \mathrm{AKT}$ signalling pathway switches the function of EZH2 from a PRC2 to a transcriptional coactivator of AR in PCa. Moreover, EZH2 may also methylate AR at lysines 630 and 632 and enhance its transcriptional activity without any synergistic relationship with polycomb repressors, suggesting that targeting more pathways including those that regulate the activity of EZH2 independent polycomb repressors would have therapeutic efficacy in suppressing PCa tumorigenesis and metastasis [44].

Other PRC2 binding partners that mediate aberrant epigenetic activities in $\mathrm{PCa}$ are bromodomain adjacent to zinc finger domain 2A (BAZ2A), JARID1A/B/D, and KDM4A. The BAZ2A, also known as TTF-1 interacting 5, represses numerous protein-coding genes that promote cell growth and proliferation. Overexpression of BAZ2A correlates with molecular subtype displaying a CpG island methylator phenotype that aberrantly alters gene expression, leading to $\mathrm{PCa}$ aggressiveness, metastasis, and recurrence [45]. BAZ2A harbours C-termini with tandem PHD finger/bromodomain that interacts with EZH2, which is a key mediator for heterochromatic histone signatures and de novo DNA methylation. Jumonji AT-rich interactive domain 1 protein (JARID1) is one of the JARID family proteins that partners with PRC2 to induce H3K4me2/3 through EZH2. JARID1 is also known to disrupt the activation of pathways such as $\mathrm{Fe}(\mathrm{II}) / \alpha$-ketoglutarate-dependent oxygenase and regulates cell survival and metastasis. Overexpression of JARID1A/B/D has been observed in PCa and metastasis, and its deletion or knockdown is associated with poor prognosis [46]. The prognostic value of JARID1D in PCa was also observed when upregulated JARID1D repressed the transcription of Snail family zinc finger 2 and miR-21-targeted matrix metallopeptidase (MMP) family genes, leading to reduced invasion and metastasis [47-49]. Regulation of AR-regulated and BTG2targeted $m i R-32$ is associated with $\mathrm{PCa}$ chemoresistance [50]. Upregulation of JMJD2A, also known as lysine-specific demethylase 4A (KDM4A), has also been observed in both human and mice models with PCa. This significantly correlates with advanced PCa and metastasis. JMJD2A appears to interact with ETS transcription factor ETV1 as well as tumour suppressor genes phosphatase and tensin homolog 
(Pten) and yes associated protein 1 (YAP1), which promotes $\mathrm{PCa}$ initiation and aggressiveness [51]. Additionally, KDM4A was demonstrated to cooperate with miRNAs in regulating gene transcription and cellular processes in $\mathrm{PCa}$, supporting an interaction between epigenetic alterations and miRNAs profiles [52].

\section{MicroRNA and Epigenetics Alterations in Prostate Cancer}

Noncoding miRNAs are evolutionary conserved short regulatory endogenous RNA molecules ( 24 nucleotides in length) that are not translated into protein even though they are transcribed from DNA. They disrupt the function of messenger RNA, leading to dysregulation in RNA silencing and gene expression at posttranscriptional and translational levels. The miRNAs may function as tumour suppressors or oncogenes and can be either downregulated or upregulated in PCa. The miRNA profiling studies demonstrate that mRNAs may act independently or in partnership with other transcription factors to regulate gene transcription, which ultimately leads to perturbed cellular processes in PCa. Several miRs in addition to those described and reviewed by Lo et al. 2013 are dysregulated in PCa [53]. The miR$101 / 31$ was found to be downregulated in metastatic PCa, affecting the expression of $\mathrm{EZH} 2$ and regulation of $\mathrm{PRC1}$ and B lymphoma Mo-MLV insertion region 1 homolog (BMI1) $[54,55]$. This promotes tumour progression by targeting AR, Stathmin 1, and cyclooxygenase-2, which were shown to be an independent prognostic indicator for recurrent PCa [55, 56]. The $m i R-24$ was demonstrated to target proapoptotic gene Fas associated factor 1 by binding to its open reading frame, thereby enhancing apoptosis in DU-145 PCa cells [57]. In other studies, suppression of p63-mediated miR-205, Frizzled7-mediated miR-613, and Myc-mediated miR-26a expression levels was observed in PCa tissues as compared to their normal counterparts $[24,58]$. Moreover, stable $m i R-26 a$ induced G1 phase arrest and epithelial mesenchymal transition (EMT), leading to significantly reduced proliferation and metastasis via activation of Wnt5a pathway [58]. Similar effects in relation to EMT regulation were observed with downregulation of the $m i R-182 / 203$ via suppression of SNAI2 and with the $m i R-144 / 145$ associated with bone, skeletal, and seminal vesicle metastases in PCa patients $[59,60]$. Genistein downregulates $m i R-205 / 31, m i R-221 / 222$, $m i R-574-36 b$, and $m i R-574-36 b$ and confers resistance to chemotherapyinduced apoptosis in PCa cells [61-63]. Demethylation of miR-146a promoter by 5 -Aza- $2^{\prime}$-deoxycytidine significantly enhanced $m i R-146 a$ expression, leading to delayed progression of castration-resistant PCa. [64].

\section{Risk Factors for Prostate Cancer and Associated Epigenetics Alterations}

A significant amount of data has shown that certain risk factors including old age, familial hereditary, and ethnicity/race may induce PCa growth by influencing both genetic and epigenetic factors [65]. Advancing age and ethnicity are the greatest nonmodifiable risk factors and play a pivotal role in PCa development (Figure 2). The risk of developing $\mathrm{PCa}$ increases exponentially after the age of 50 years [66]. Seminal work published by Kwabi-Addu and his coauthors in the Clinical Cancer Research showed that methylation of GSTPi, RARß2, RASSF1A, NK2 homeobox 5 (NKX-2-5), and estrogen receptor 1 (ESR1) tumours suppressor genes in prostate tissues is age-independent [67]. Methylation of these genes was shown to initiate in normal prostate tissues as old age strikes and markedly increases during the progression of PCa [67]. Age-related CpG island methylation is significantly enriched with DNA binding and transcriptional factors [68]. Jung et al. and his team in Van Andel Research Institute are currently investigating the epigenomic changes following environmental factors including aging. They developed the methylated $\mathrm{CpG}$ island recovery assay, which is a reliable method for comparing genome-wide DNA methylations in normal and cancer tissues [69]. More recently, the same group hypothesised that polycomb complex resulting from KDM2B interaction with $\mathrm{PRC1} / 2$ that recognize unmethylated $\mathrm{CpG}$ islands may degrade with age. This would, in turn, allow access of CpG islands to the DNMT3A and DNMT3B, leading to partial DNA methylation in aging men [70].

The risk of PCa is increased in black African and American men compared to Caucasian men, suggesting an important role of ethnicity/race in PCa development [71]. Several studies showed that black South African men present with higher PSA levels and more aggressive and metastatic PCa than black American men. The presence of these phenotypes correlates with socioeconomic status, poor $\mathrm{PCa}$ awareness, and screening facilities associated with developing countries $[66,71]$. Racial/ethnic disparities in epigenetic alterations have been observed in PCa tissues and correlate with racial differences in cancer prognosis and survival [72]. Devaney et al. [73] revealed that genome-wide methylation profiles differ in the PCa tissues between African-American and Caucasian men. However, considerable research investigations are still required to elucidate the role of these methylation differences in PCa disparity.

Similar to most cancers, $\mathrm{PCa}$ is a genetic disease that can also be caused by both germline and somatic mutations. Family hereditary has been postulated to increase the risk of PCa development. Men older than 55 years who have a first-degree male relative (i.e., father, son, and brother) diagnosed with $\mathrm{PCa}$ are at higher risk of acquiring $\mathrm{PCa}$ [74]. Genetic susceptibility and aberrant epigenetics may predispose individuals to $\mathrm{PCa}$ [65]. For instance, mutations or abnormal gene expression associated with classic genes such as $p 53, P T E N, B R C A 1$, and BRCA2 may contribute to PCa development and metastasis [75-77]. Epidemiological studies have also indicated that male carriers of BRCA1 and BRCA2 mutations have the highest risk of developing PCa compared to noncarriers of these mutations. This coincides with more aggressive disease and low rates of survival $[78,79]$.

Diet and environmental and occupational factors are hypothesised to be modifiable PCa-related risk factors. 
Nonmodifiable risk factors

Old age ( $>50$ years)

(i) Increased promoter methylation of GSTP $i$, RAR 32 , RASSF1A, NKX-2-5, and ESR1 correlates with aging.
Ethnicity/race

(i) Black men $>$ Caucasian men

(ii) Methylation profiles differ in the PCa tissues between African-American and Caucasian men.

(i) Father/and brother

(ii) Mutations or abnormal gene expressions associated with $p 53, P T E N, B R C A 1$, and BRCA2 tumour suppressor genes have been observed in PCa.

Modifiable risk factors (i) Exposure to polychlorinated biphenyls, polycyclic aromatic hydrocarbons, herbicides, and pesticides is postulated to increase the risk of developing PCa.

FIGURE 2: Risk factors associated with the development of prostate cancer. PCa risk factors can be categorised as nonmodifiable (e.g., old age, ethnicity/race, and familial hereditary) and modifiable (diet and environmental/occupational factors).

However, the mechanisms underlying the link of these factors and PCa development remain unclear $[80,81]$. The $\alpha$ methylacyl-CoA racemase is a peroxisomal enzyme required for the oxidation of branched-chain trans-fatty acids from red meat and dairy products. This enzyme was found to be aberrantly upregulated in PCa cells, supporting the hypothesis that a diet high in saturated or trans fats is associated with increased risk for PCa development [82, 83]. Certain diets containing antioxidant or anticancer properties such as genistein, resveratrol, epigallocatechin-3-gallate (polyphenols), isothiocyanates, folate, zinc, curcumin, and mono- or polyunsaturated fatty acids may serve as protective factors for PCa (Figure 2). Some of these anticancer agents were found to inhibit tumour proliferation and promote apoptosis by suppressing trimethylation of H3K27 through PRC2 arrest $[84,85]$. Genistein is an important nutraceutical compound derived from soy products and has been shown to confer protection from developing cancer. Genistein suppresses multiple cellular processes such as proliferation and angiogenesis by demethylating tumour suppressor genes p16, MGMT, GSTPi, RAR $\beta$, and $h M L H 1$. Rajvir Dahiya and his colleagues from University of Southern California at San Francisco have recently demonstrated that genistein regulates various miRs including $m i R$ - $1260 b$, leading to the upregulation of $s F R P 1$ and Smad4 in PCa cell lines via DNA demethylation and histone modifications, suggesting that diet may also prompt epigenetic alterations and contribute to PCa development [86].

\section{Epigenetic Therapy in Prostate Cancer}

Substantial knowledge on abnormal epigenetic alterations and cancer development is now being translated into novel anticancer therapeutic approaches [87, 88]. Unlike genetic mutations, aberrant epigenetic alterations are potentially pharmaceutically reversible making them attractive targets for chemotherapeutic approaches. DNMTs, HDACs, EZH2, DOT1-like histone H3K79 methyltransferase (DOT1L), and bromodomain and extraterminal (BET) are the current targets of epigenetic therapies (Figure 3). Inhibitors against these epigenetic mediators emerged as promising chemotherapeutic agents in several cancer models $[87,89]$.

Available demethylating agents that inhibit the action of DNMT include nucleoside (5-azacytidine, 5-azacytidine$2^{\prime}$-deoxycytidine/decitabine, and zebularine) and nonnucleoside (procaine, procainamide, disulfiram, and RG108) inhibitors [88]. These inhibitors have exhibited efficient antitumorigenic activities by suppressing an altered expression of tumour suppressor genes in haematological malignancies, breast, gastric, lung, ovarian, and hepatocellular carcinomas. Convincing clinical evidence of most DNMT inhibitors in PCa is still lacking and remains elusive. However, the epigenetic therapeutic potential of decitabine, procainamide, disulfiram, and RG108 was recently observed in human PCa cell lines and xenograft models, correlating with significant reduction in tumour growth and increased apoptosis [87-91]. 

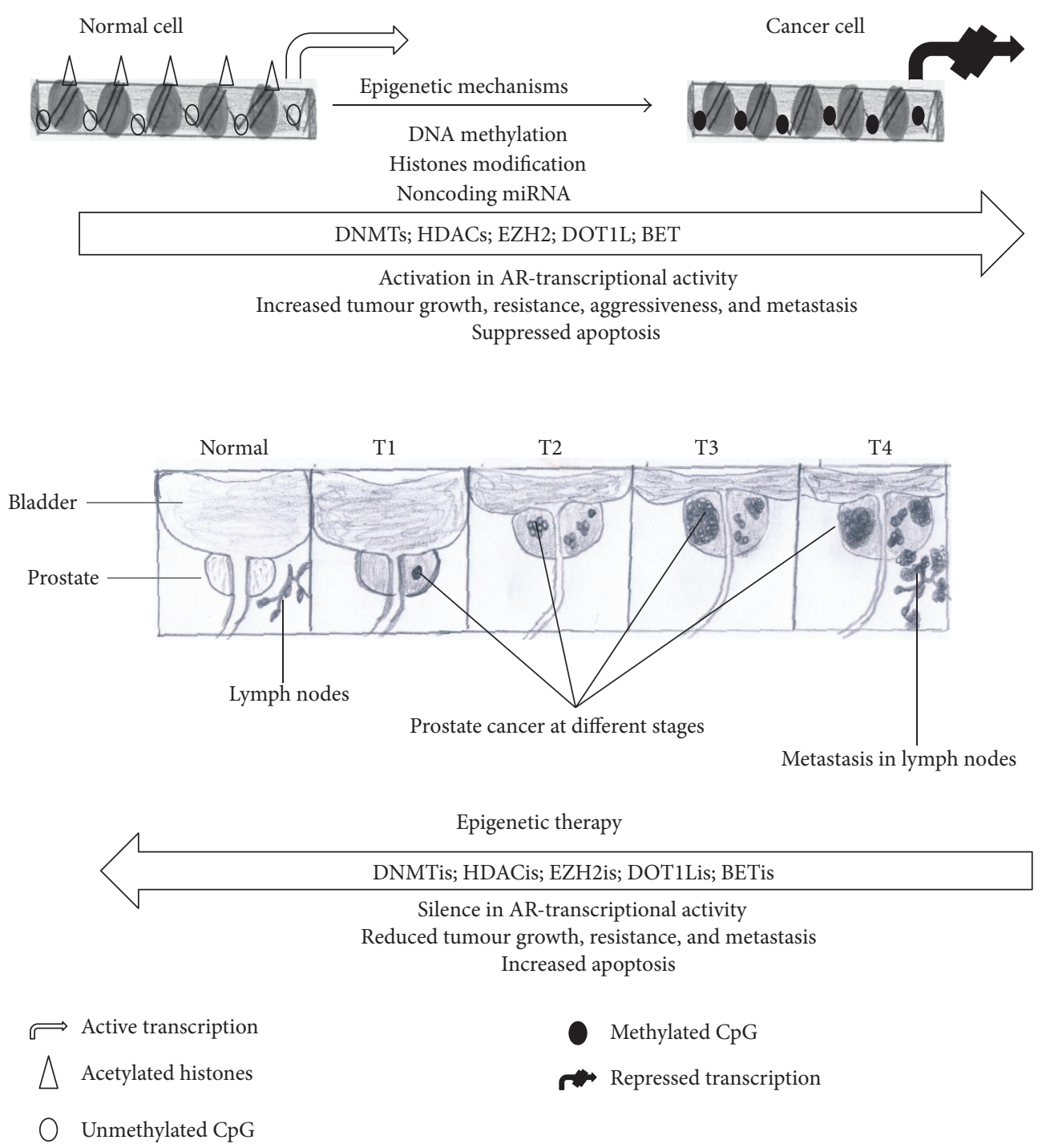

Methylated CpG
$\rightarrow \sim$ Repressed transcription

FIGURE 3: Epigenetic mechanisms and therapy in advanced prostate cancer. Prostate cancer follows aberrant epigenetic alterations that are associated with perturbed cellular processes that are critical in tumorigenesis. In normal cell, CpG islands are protected from DNA methylation and deacetylation whereas a prostate cancer cell is characterised by tumour growth, differentiation, resistance, and metastasis resulting from aberrant DNA methylation and deacetylation. AR: androgen receptor; BET: bromodomain and extraterminal; CpG: cytosinephosphate-guanine island; DNMTs: DNA methyltransferases; DOT1L: DOT1-like histone H3K79 methyltransferase; EZH2: enhancer of zeste 1; HDACs: histone deacetylases; DNMTs: DNA methyltransferase inhibitors; HDACs: histone deacetylase inhibitors.

HDAC inhibitors (HDACis) are class of anticancer agents that reverse autonomous epigenetic alterations by disrupting the activity of HDACs, and their action coincides with sustained apoptosis resulting from impeded cell differentiation, angiogenesis, and metastasis. Vorinostat and romidepsin are the first FDA and EMEA approved HDACis and are currently used to treat uncontrolled cutaneous $\mathrm{T}$ cell lymphoma. Vorinostat has been investigated extensively in biologic, preclinical, and clinical studies involving several cancers and has emerged as a promising antitumor and antimetastatic agent with a favourable safety profile. Treatment with vorinostat represses PC-3 xenograft tumours and inhibits cell proliferation and metastasis in PC-3, LNCaP, and DU-145 human PCa cell lines [92]. Similar effects were observed in vorinostattreated mice transplanted with CWR222 PCa tumours [93]. Romidepsin disrupts the interaction between Hsp90 and its binding partners, thereby inducing the Hsp90 hyperacetylation and abrogation of AR signalling. In castration-resistant PCa clinical models, romidepsin correlates with minimal clinical activity. Other new HDACis that are currently undergoing investigation in PCa cell line models include panobinostat, MCL33(S)-2, and MHY219 [94, 95]. Combination therapy of vorinostat and romidepsin with other chemotherapeutic drugs including demethylating agents was shown to have more maximal activity than monotherapy and synergistically enhanced apoptosis in PCa cell lines ALVA-31, 
LNCaP, and DU-154 [96, 97]. Recently, a new hybrid of vorinostat and a topoisomerase inhibitor WJ3543 exhibited potential antitumor activities, which overlapped with repressed cell growth, proliferation, and increased apoptosis in metastatic PCa [98].

While the efficacy of most lysine methyltransferases inhibitors in PCa remains obscure, there has been some advancement in preclinical development and phase 1 clinical studies with inhibitors targeting EZH2, DOT1L, and BET. BET inhibitor I-BET762 potentially suppressed cell growth and tumour aggressiveness in vivo by reducing MYC expression in PCa cancer cell lines, xenograft mouse model, and human tissues [99, 100]. EZH2 inhibitors GlaxoSmithKline-126 (GSK-126) and 3-deazaneplanocin-A reduced AR-transcriptional activity, leading to the inhibition of cancer cells self-renewal mechanisms, tumorigenesis, and metastasis mediated by aberrant EZH2 upregulation in PCa cells $[101,102]$. Furthermore, combinations of nontoxic topoisomerase poisons VP-16 and GSK-126 significantly enhanced apoptosis in in vitro viability assays [102]. Other EZH2 inhibitors such as EPZ005667, GSK343, EP2-6436, Novartis, and EIl also demonstrated their chemotherapeutic potential in treating several malignancies. Anti-DOT1L inhibitors such as EPZ004777, EPZ003696, EPZ5676, Yao CMP4, BrSAH, and SGC946 have been identified and tested in leukemic cells, where they were associated with suppression of H3K27 and tumour growth [103-107]. These inhibitors and other EZH2 inhibitors including EPZ005667, GSK343, EP2-6436, Novartis, and EIl warrant clinical investigations in PCa.

\section{Conclusion}

Current evidence supports previous studies and demonstrates that epigenetic mechanisms play a critical role in PCa development. PCa-related epigenetic alterations may be triggered by induced genomic instabilities as well as various modifiable and nonmodifiable risk factors. Aberrant DNA methylation, histone modification, and noncoding RNA are tightly linked and synergistically alter gene transcription and normal gene function. These mechanisms lead to perturbed cellular pathways that are associated with abnormal or pathological phenotypes in prostate gland. Although several transcriptional factors have been demonstrated to interact with epigenetic regulators and modifiers, the underlying mechanisms are clearly complex and remain elusive. In terms of clinical application, epigenetic alterations in PCA are also potentially pharmacologically reversible. However, more preclinical and clinical studies are still needed to explore the chemotherapeutic potential of demethylation agents including EZH2, DOT1L, and BET. This may enable the development of more novel chemotherapeutic drugs that will be used in conjunction with current conventional treatments such as ${ }^{77} \mathrm{Lu}$-PSMA-RLT to improve the management of refractory and metastasised PCa.

\section{Competing Interests}

The authors declare no conflict of interests.

\section{Acknowledgments}

This work was supported by grants from Oppenheimer Memorial Trust, Poliomyelitis Research Foundation, and National Research Foundation.

\section{References}

[1] S. J. Fourie, A. McMaster, R. Mothilal, and K. I. Maart, "A phase IV clinical trial of patients with solid tumors receiving lenog rastim as primary prophylaxis for chemotherapy-induced neutropenia, in a docetaxel-based regimen," Journal of Cancer Research, vol. 2014, Article ID 684936, 7 pages, 2014.

[2] G. Alleyne, A. Binagwaho, A. Haines et al., "Embedding noncommunicable diseases in the post-2015 development agenda," The Lancet, vol. 381, no. 9866, pp. 566-574, 2013.

[3] R. Siegel, D. Naishadham, and A. Jemal, "Cancer statistics, 2013," CA Cancer Journal for Clinicians, vol. 63, no. 1, pp. 11-30, 2013.

[4] W. Catalona, J. Richie, F. Ahmann et al., "In reply: re comparison of prostate specific antigen concentration versus prostate specific antigen density in the early detection of prostate cancer receiver operating characteristic curves; re selection of optimal prostate specific antigen cutoffs for early detection of prostate cancer receiver operating characteristic curves," The Journal of Urology, vol. 154, pp. 1145-1146, 1995.

[5] M. J. Barry, "Screening for prostate cancer-the controversy that refuses to die," New England Journal of Medicine, vol. 360, no. 13, pp. 1351-1354, 2009.

[6] B. A. Lipski, R. L. Garcia, and M. K. Brawer, "Prostatic intraepithelial neoplasia: significance and management," Seminars in Urologic Oncology, vol. 14, no. 3, pp. 149-155, 1996.

[7] J. I. Epstein, M. J. Zelefsky, D. D. Sjoberg et al., "A contemporary prostate cancer grading system: a validated alternative to the gleason score," European Urology, vol. 69, pp. 428-435, 2016.

[8] A. O. Sartor, "Progression of metastatic castrate-resistant prostate cancer: impact of therapeutic intervention in the postdocetaxel space," Journal of Hematology \& Oncology, vol. 4, article 18, 2011.

[9] T. A. Skolarus, A. M. D. Wolf, N. L. Erb et al., "American Cancer Society prostate cancer survivorship care guidelines," $C A$ : Cancer Journal for Clinicians, vol. 64, no. 4, pp. 225-249, 2014.

[10] H. Hieronymus, N. Schultz, A. Gopalan et al., "Copy number alteration burden predicts prostate cancer relapse," Proceedings of the National Academy of Sciences of the United States of America, vol. 111, no. 30, pp. 11139-11144, 2014.

[11] E. D. Crawford, C. S. Higano, N. D. Shore, M. Hussain, and D. P. Petrylak, "Treating patients with metastatic castration resistant prostate cancer: a comprehensive review of available therapies," The Journal of Urology, vol. 194, no. 6, pp. 1537-1547, 2015.

[12] A. Afshar-Oromieh, E. Avtzi, F. L. Giesel et al., "The diagnostic value of PET/CT imaging with the ${ }^{68} \mathrm{Ga}$-labelled PSMA ligand HBED-CC in the diagnosis of recurrent prostate cancer," European Journal of Nuclear Medicine and Molecular Imaging, vol. 42, no. 2, pp. 197-209, 2015.

[13] D. A. Silver, I. Pellicer, W. R. Fair, W. D. W. Heston, and C. Cordon-Cardo, "Prostate-specific membrane antigen expression in normal and malignant human tissues," Clinical Cancer Research, vol. 3, no. 1, pp. 81-85, 1997.

[14] C. Kratochwil, F. L. Giesel, M. Stefanova et al., "PSMA-targeted radionuclide therapy of metastatic castration-resistant prostate cancer with 177Lu-labeled PSMA-617," Journal of Nuclear Medicine, vol. 57, no. 8, pp. 1170-1176, 2016. 
[15] R. P. Baum, H. R. Kulkarni, C. Schuchardt et al., "177 Lulabeled prostate-specific membrane antigen radioligand therapy of metastatic castration-resistant prostate cancer: safety and efficacy," Journal of Nuclear Medicine, vol. 57, no. 7, pp. 10061013, 2016.

[16] G. J. Kelloff, P. Choyke, and D. S. Coffey, "Challenges in clinical prostate cancer: role of imaging," American Journal of Roentgenology, vol. 192, no. 6, pp. 1455-1470, 2009.

[17] P. Lambin, E. Rios-Velazquez, R. Leijenaar et al., "Radiomics: extracting more information from medical images using advanced feature analysis," European Journal of Cancer, vol. 48, no. 4, pp. 441-446, 2012.

[18] A. Rajinikanth, M. Manoharan, C. T. Soloway, F. J. Civantos, and M. S. Soloway, "Trends in Gleason score: concordance between biopsy and prostatectomy over 15 years," Urology, vol. 72, no. 1, pp. 177-182, 2008.

[19] M. Maio, A. Covre, E. Fratta et al., "Molecular pathways: at the crossroads of cancer epigenetics and immunotherapy," Clinical Cancer Research, vol. 21, no. 18, pp. 4040-4047, 2015.

[20] A. J. Christiansen, A. West, K.-M. Banks et al., "Eradication of solid tumors using histone deacetylase inhibitors combined with immune-stimulating antibodies," Proceedings of the National Academy of Sciences of the United States of America, vol. 108, no. 10, pp. 4141-4146, 2011.

[21] P. A. Jones and S. B. Baylin, "The fundamental role of epigenetic events in cancer," Nature Reviews Genetics, vol. 3, no. 6, pp. 415428, 2002.

[22] G. Egger, G. Liang, A. Aparicio, and P. A. Jones, "Epigenetics in human disease and prospects for epigenetic therapy," Nature, vol. 429, no. 6990, pp. 457-463, 2004.

[23] Z. Kote-Jarai, C. Mikropoulos, D. A. Leongamornlert et al., "Prevalence of the HOXB13 G84E germline mutation in British men and correlation with prostate cancer risk, tumour characteristics and clinical outcomes," Annals of Oncology, vol. 26, no. 4, pp. 756-761, 2015.

[24] W. Ren, C. Li, W. Duan et al., "MicroRNA-613 represses prostate cancer cell proliferation and invasion through targeting Frizzled7," Biochemical and Biophysical Research Communications, vol. 469, no. 3, pp. 633-638, 2016.

[25] K. D. Robertson, "DNA methylation and chromatinunraveling the tangled web," Oncogene, vol. 21, no. 35, pp. 5361-5379, 2002.

[26] L.-C. Li, P. R. Carroll, and R. Dahiya, "Epigenetic changes in prostate cancer: implication for diagnosis and treatment," Journal of the National Cancer Institute, vol. 97, no. 2, pp. 103-115, 2005.

[27] D. E. Linn, K. L. Penney, R. T. Bronson, L. A. Mucci, and Z. $\mathrm{Li}$, "Deletion of interstitial genes between TMPRSS2 and ERG promotes prostate cancer progression," Cancer Research, vol. 76, no. 7, pp. 1869-1881, 2016.

[28] M. Chu, Y. Chang, N. Wang, W. Li, P. Li, and W.-Q. Gao, "Hypermethylation-mediated transcriptional repression of TMPRSS2 in androgen receptor-negative prostate cancer cells," Experimental Biology and Medicine, vol. 239, no. 7, pp. 823-828, 2014.

[29] B. G. Barwick, M. Abramovitz, M. Kodani et al., "Prostate cancer genes associated with TMPRSS2-ERG gene fusion and prognostic of biochemical recurrence in multiple cohorts," British Journal of Cancer, vol. 102, no. 3, pp. 570-576, 2010.

[30] S. A. Tomlins, B. Laxman, S. Varambally et al., "Role of the TMPRSS2-ERG gene fusion in prostate cancer," Neoplasia, vol. 10, no. 2, pp. 177-188, 2008.
[31] S. A. Tomlins, D. R. Rhodes, S. Perner et al., "Recurrent fusion of TMPRSS2 and ETS transcription factor genes in prostate cancer," Science, vol. 310, no. 5748, pp. 644-648, 2005.

[32] R. Zelic, V. Fiano, C. Grasso et al., "Global DNA hypomethylation in prostate cancer development and progression: a systematic review," Prostate Cancer and Prostatic Diseases, vol. 18, no. 1, pp. 1-12, 2015.

[33] H. Kinoshita, Y. Shi, C. Sandefur et al., "Methylation of the androgen receptor minimal promoter silences transcription in human prostate cancer," Cancer Research, vol. 60, no. 13, pp. 3623-3630, 2000.

[34] D. K. Mishra, Z. Chen, Y. Wu, M. Sarkissyan, H. P. Koeffler, and J. V. Vadgama, "Global methylation pattern of genes in androgen-sensitive and androgen-independent prostate cancer cells," Molecular Cancer Therapeutics, vol. 9, no. 1, pp. 33-45, 2010.

[35] M. M. Pomerantz, F. Li, D. Y. Takeda et al., "The androgen receptor cistrome is extensively reprogrammed in human prostate tumorigenesis," Nature Genetics, vol. 47, no. 11, pp. 1346-1351, 2015.

[36] Y. Chen, P. Chi, S. Rockowitz et al., "ETS factors reprogram the androgen receptor cistrome and prime prostate tumorigenesis in response to PTEN loss," Nature Medicine, vol. 19, no. 8, pp. 1023-1029, 2013.

[37] K.-I. Takayama, A. Misawa, T. Suzuki et al., “TET2 repression by androgen hormone regulates global hydroxymethylation status and prostate cancer progression," Nature Communications, vol. 6, article 8219, 2015.

[38] X.-J. Yang and E. Seto, "Lysine acetylation: codified crosstalk with other posttranslational modifications," Molecular Cell, vol. 31, no. 4, pp. 449-461, 2008.

[39] L. Ringrose and R. Paro, "Epigenetic regulation of cellular memory by the polycomb and trithorax group proteins," Annual Review of Genetics, vol. 38, pp. 413-443, 2004.

[40] S. Varambally, S. M. Dhanasekaran, M. Zhou et al., "The polycomb group protein EZH2 is involved in progression of prostate cancer," Nature, vol. 419, no. 6907, pp. 624-629, 2002.

[41] P. L. Clermont, D. Lin, F. Crea et al., "Polycomb-mediated silencing in neuroendocrine prostate cancer," Clinical Epigenetics, vol. 7, article 40, 2015.

[42] G. P. Souroullas, W. R. Jeck, J. S. Parker et al., "An oncogenic Ezh2 mutation induces tumors through global redistribution of histone 3 lysine 27 trimethylation," Nature Medicine, vol. 22, no. 6, pp. 632-640, 2016.

[43] L. Jiao and X. Liu, "Structural basis of histone H3K27 trimethylation by an active polycomb repressive complex 2," Science, vol. 350, no. 6258, article aac4383, 2015.

[44] K. Xu, Z. J. Wu, A. C. Groner et al., "EZH2 oncogenic activity in castration-resistant prostate cancer cells is polycombindependent," Science, vol. 338, no. 6113, pp. 1465-1469, 2012.

[45] L. Gu, S. C. Frommel, C. C. Oakes et al., "BAZ2A (TIP5) is involved in epigenetic alterations in Prostate cancer and its overexpression predicts disease recurrence," Nature Genetics, vol. 47, no. 1, pp. 22-30, 2015.

[46] J. Qi, K. Nakayama, R. D. Cardiff et al., "Siah2-dependent concerted activity of HIF and FoxA2 regulates formation of neuroendocrine phenotype and neuroendocrine prostate tumors," Cancer Cell, vol. 18, no. 1, pp. 23-38, 2010.

[47] N. Li, S. S. Dhar, T. Y. Chen et al., "JARID1D is a suppressor and prognostic marker of prostate cancer invasion and metastasis," Cancer Research, vol. 76, no. 4, pp. 831-843, 2016. 
[48] J. Sayegh, J. Cao, M. R. Zou et al., "Identification of small molecule inhibitors of Jumonji AT-rich interactive domain $1 \mathrm{~B}$ (JARID1B) histone demethylase by a sensitive high throughput screen," Journal of Biological Chemistry, vol. 288, no. 13, pp. 9408-9417, 2013.

[49] S. T. Reis, J. Pontes-Junior, A. A. Antunes et al., "miR-21 may acts as an oncomir by targeting RECK, a matrix metalloproteinase regulator, in prostate cancer," BMC Urology, vol. 12, no. 1, article 14, 2012.

[50] S. E. Jalava, A. Urbanucci, L. Latonen et al., "Androgenregulated miR-32 targets BTG2 and is overexpressed in castration-resistant prostate cancer," Oncogene, vol. 31, no. 41, pp. 4460-4471, 2012.

[51] S. Kaneko, R. Bonasio, R. Saldaña-Meyer et al., "Interactions between JARID2 and noncoding RNAs regulate PRC2 recruitment to chromatin," Molecular Cell, vol. 53, no. 2, pp. 290-300, 2014.

[52] S. N. Kamdar, L. T. Ho, K. J. Kron et al., "Dynamic interplay between locus-specific DNA methylation and hydroxymethylation regulates distinct biological pathways in prostate carcinogenesis," Clinical Epigenetics, vol. 8, article 32, 2016.

[53] U. Lo, D. Yang, and J. Hsieh, "The role of microRNAs in prostate cancer progression," Translational Andrology and Urology, vol. 2, no. 3, pp. 228-241, 2013.

[54] Q. Zhang, S. K. R. Padi, D. J. Tindall, and B. Guo, "Polycomb protein EZH2 suppresses apoptosis by silencing the proapoptotic miR-31," Cell Death and Disease, vol. 5, no. 10, article el486, 2014.

[55] P. Cao, Z. Deng, M. Wan et al., "MicroRNA-101 negatively regulates Ezh2 and its expression is modulated by androgen receptor and HIF-1 $\alpha /$ HIF-1 $\beta$," Molecular Cancer, vol. 9, no. 1, article 108, 2010.

[56] M. R. M. Roduan, N. Mohtarrudin, C. P. Pei, M. Osman, and N. M. Dusa, "High expression of cyclooxygenase-2 in high grade human prostate adenocarcinoma," Sains Malaysiana, vol. 44, no. 5, pp. 727-733, 2015.

[57] W. Qin, Y. Shi, B. Zhao et al., "miR-24 regulates apoptosis by targeting the Open Reading Frame (ORF) region of FAF1 in cancer cells," PLoS ONE, vol. 5, no. 2, article e9429, 2010.

[58] S. Zhao, X. Ye, L. Xiao et al., "MiR-26a inhibits prostate cancer progression by repression of Wnt5a," Tumor Biology, vol. 35, no. 10, pp. 9725-9733, 2014.

[59] Y. Qu, W. Li, M. R. Hellem et al., "MiR-182 and miR-203 induce mesenchymal to epithelial transition and self-sufficiency of growth signals via repressing SNAI2 in prostate cells," International Journal of Cancer, vol. 133, no. 3, pp. 544-555, 2013.

[60] X. Peng, W. Guo, T. Liu et al., "Identification of miRs-143 and -145 that is associated with bone metastasis of prostate cancer and involved in the regulation of EMT," PLOS ONE, vol. 6, no. 5, article e20341, 2011.

[61] N. Bhatnagar, X. Li, S. K. R. Padi, Q. Zhang, M.-S. Tang, and B. Guo, "Downregulation of miR-205 and miR-31 confers resistance to chemotherapy-induced apoptosis in prostate cancer cells," Cell Death \& Disease, vol. 1, no. 12, article e105, 2010.

[62] Y. Chen, M. S. Zaman, G. Deng et al., "MicroRNAs 221/222 and genistein-mediated regulation of ARHI tumor suppressor gene in prostate cancer," Cancer Prevention Research, vol. 4, no. 1, pp. 76-86, 2011.

[63] T. Chiyomaru, S. Yamamura, S. Fukuhara et al., "Genistein up-regulates tumor suppressor MicroRNA-574-3p in prostate cancer," PLoS ONE, vol. 8, no. 3, Article ID e58929, 2013.
[64] X. Wang, H. Gao, L. Ren, J. Gu, Y. Zhang, and Y. Zhang, "Demethylation of the miR-146a promoter by 5-Aza-2'-deoxycytidine correlates with delayed progression of castrationresistant prostate cancer," BMC Cancer, vol. 14, no. 1, article 308, 2014.

[65] D. Karan, J. Thrasher, and D. Lubaroff, "Prostate cancer: genes, environment, immunity and the use of immunotherapy," Prostate Cancer and Prostatic Diseases, vol. 11, no. 3, pp. 230-236, 2008.

[66] C. F. Heyns, M. Fisher, A. Lecuona, and A. van der Merwe, "Prostate cancer among different racial groups in the western cape: presenting features and management," South African Medical Journal, vol. 101, no. 4, pp. 267-270, 2011.

[67] B. Kwabi-Addo, W. Chung, L. Shen et al., "Age-related DNA methylation changes in normal human prostate tissues," Clinical Cancer Research, vol. 13, no. 13, pp. 3796-3802, 2007.

[68] T. Yuan, Y. Jiao, S. de Jong, R. A. Ophoff, S. Beck, and A. E. Teschendorff, "An integrative multi-scale analysis of the dynamic DNA methylation landscape in aging," PLoS Genetics, vol. 11, no. 2, Article ID e1004996, 2015.

[69] M. Jung, S. Kadam, W. Xiong, T. A. Rauch, S.-G. Jin, and G. P. Pfeifer, "MIRA-seq for DNA methylation analysis of CpG islands," Epigenomics, vol. 7, no. 5, pp. 695-706, 2015.

[70] M. Jung and G. P. Pfeifer, "Aging and DNA methylation," BMC Biology, vol. 13, no. 1, 2015.

[71] N. Mofolo, O. Betshu, O. Kenna et al., "Knowledge of prostate cancer among males attending a urology clinic, a South African study," SpringerPlus, vol. 4, article 67, 2015.

[72] Y.-Y. Xia, Y.-B. Ding, X.-Q. Liu et al., "Racial/ethnic disparities in human DNA methylation," Biochimica et Biophysica ActaReviews on Cancer, vol. 1846, no. 1, pp. 258-262, 2014.

[73] J. M. Devaney, S. Wang, P. Furbert-Harris et al., "Genome-wide differentially methylated genes in prostate cancer tissues from African-American and Caucasian men," Epigenetics, vol. 10, no. 4, pp. 319-328, 2015.

[74] A. Bjartell, "Genetic markers and the risk of developing prostate cancer," European Urology, vol. 60, no. 1, pp. 29-31, 2011.

[75] E. Castro and R. Eeles, "The role of BRCA1 and BRCA2 in prostate cancer," Asian Journal of Andrology, vol. 14, no. 3, pp. 409-414, 2012.

[76] N. Rivlin, R. Brosh, M. Oren, and V. Rotter, "Mutations in the p53 tumor suppressor gene: important milestones at the various steps of tumorigenesis," Genes \& Cancer, vol. 2, no. 4, pp. 466474, 2011.

[77] C. E. Barbieri, C. H. Bangma, A. Bjartell et al., "The mutational landscape of prostate cancer," European Urology, vol. 64, no. 4, pp. 567-576, 2013.

[78] H. Cavanagh and K. M. A. Rogers, "The role of BRCA1 and BRCA2 mutations in prostate, pancreatic and stomach cancers," Hereditary Cancer in Clinical Practice, vol. 13, no. 1, article 16, pp. 1-7, 2015.

[79] C. Rosty, M. D. Walsh, N. M. Lindor et al., "High prevalence of mismatch repair deficiency in prostate cancers diagnosed in mismatch repair gene mutation carriers from the colon cancer family registry," Familial Cancer, vol. 13, no. 4, pp. 573-582, 2014.

[80] J. Peto, "Cancer epidemiology in the last century and the next decade," Nature, vol. 411, no. 6835, pp. 390-395, 2001.

[81] H. Shimizu, R. K. Ross, L. Bernstein, R. Yatani, B. E. Henderson, and T. M. Mack, "Cancers of the prostate and breast among Japanese and white immigrants in Los Angeles County," British Journal of Cancer, vol. 63, no. 6, pp. 963-966, 1991. 
[82] M. D. Lloyd, M. Yevglevskis, G. L. Lee, P. J. Wood, M. D. Threadgill, and T. J. Woodman, " $\alpha$-methylacyl-CoA racemase (AMACR): metabolic enzyme, drug metabolizer and cancer marker P504S," Progress in Lipid Research, vol. 52, no. 2, pp. 220230, 2013.

[83] Y. Liu, "Fatty acid oxidation is a dominant bioenergetic pathway in prostate cancer," Prostate Cancer and Prostatic Diseases, vol. 9, no. 3, pp. 230-234, 2006.

[84] L. J. Gudas and J. A. Wagner, "Retinoids regulate stem cell differentiation," Journal of Cellular Physiology, vol. 226, no. 2, pp. 322-330, 2011.

[85] A. P. Bracken and K. Helin, "Polycomb group proteins: navigators of lineage pathways led astray in cancer," Nature Reviews Cancer, vol. 9, no. 11, pp. 773-784, 2009.

[86] H. Hirata, K. Ueno, K. Nakajima et al., "Genistein downregulates onco-miR-1260b and inhibits Wnt-signalling in renal cancer cells," British Journal of Cancer, vol. 108, no. 10, pp. 20702078, 2013.

[87] I. Naldi, M. Taranta, L. Gherardini et al., "Novel epigenetic target therapy for prostate cancer: a preclinical study," PLoS ONE, vol. 9, no. 5, Article ID e98101, 2014.

[88] I. Graça, E. J. Sousa, T. Baptista et al., "Anti-tumoral effect of the non-nucleoside DNMT inhibitor RG108 in human prostate cancer cells," Current Pharmaceutical Design, vol. 20, no. 11, pp. 1803-1811, 2014.

[89] M. Karahoca and R. L. Momparler, "Pharmacokinetic and pharmacodynamic analysis of 5-aza-2' - deoxycytidine (decitabine) in the design of its dose-schedule for cancer therapy," Clinical Epigenetics, vol. 5, no. 1, article 3, 2013.

[90] A. Hagelgans, M. Menschikowski, S. Fuessel et al., "Deregulated expression of urokinase and its inhibitor type 1 in prostate cancer cells: role of epigenetic mechanisms," Experimental and Molecular Pathology, vol. 94, no. 3, pp. 458-465, 2013.

[91] B. Fialova, K. S. Trtkova, L. Paskova, K. Langova, and Z. Kolar, "Effect of histone deacetylase and DNA methyltransferase inhibitors on the expression of the androgen receptor gene in androgen-independent prostate cancer cell lines," Oncology Reports, vol. 29, no. 5, pp. 2039-2045, 2013.

[92] S. K. Kulp, C. S. Chen, D. S. Wang, C. Y. Chen, and C. S. Chen, "Antitumor effects of a novel phenylbutyrate-based histone deacetylase inhibitor, (S)-HDAC-42, in prostate cancer," Clinical Cancer Research, vol. 12, no. 17, pp. 5199-5206, 2006.

[93] L. M. Butler, D. B. Agus, H. I. Scher et al., "Suberoylanilide hydroxamic acid, an inhibitor of histone deacetylase, suppresses the growth of prostate cancer cells in vitro and in vivo," Cancer Research, vol. 60, no. 18, pp. 5165-5170, 2000.

[94] A. Laurenzana, M. Balliu, C. Cellai, M. N. Romanelli, and F. Paoletti, "Effectiveness of the histone deacetylase inhibitor (S)2 against LNCaP and PC3 human prostate cancer cells," PLoS ONE, vol. 8, no. 3, Article ID e58267, 2013.

[95] N. Patra, U. De, T. H. Kim et al., "A novel histone deacetylase (HDAC) inhibitor MHY219 induces apoptosis via upregulation of androgen receptor expression in human prostate cancer cells," Biomedicine and Pharmacotherapy, vol. 67, no. 5, pp. 407-415, 2013.

[96] V. Lakshmikanthan, I. Kaddour-Djebbar, R. W. Lewis, and M. V. Kumar, "SAHA-sensitized prostate cancer cells to TNF $\alpha$-related apoptosis-inducing ligand (TRAIL): mechanisms leading to synergistic apoptosis," International Journal of Cancer, vol. 119, no. 1, pp. 221-228, 2006.
[97] R. L. VanOosten, J. K. Earel Jr., and T. S. Griffith, "Histone deacetylase inhibitors enhance Ad5-TRAIL killing of TRAILresistant prostate tumor cells through increased caspase-2 activity," Apoptosis, vol. 12, no. 3, pp. 561-571, 2007.

[98] C. Yu, S. Pan, S. Chao et al., "A novel small molecule hybrid of vorinostat and DACA displays anticancer activity against human hormone-refractory metastatic prostate cancer through dual inhibition of histone deacetylase and topoisomerase I," Biochemical Pharmacology, vol. 90, no. 3, pp. 320-330, 2014.

[99] I. A. Asangani, V. L. Dommeti, X. Wang et al., "Therapeutic targeting of BET bromodomain proteins in castration-resistant prostate cancer," Nature, vol. 510, no. 7504, pp. 278-282, 2014.

[100] A. Wyce, Y. Degenhardt, Y. Bai et al., "Inhibition of BET bromodomain proteins as a therapeutic approach in prostate cancer," Oncotarget, vol. 4, no. 12, pp. 2419-2429, 2013.

[101] F. Crea, E. M. Hurt, L. A. Mathews et al., "Pharmacologic disruption of Polycomb Repressive Complex 2 inhibits tumorigenicity and tumor progression in prostate cancer," Molecular Cancer, vol. 10, article 40, 2011.

[102] K. F. Schaarschuch, Co-Regulators of Androgen Receptor Transcriptional Activity as a Novel Therapeutic Target for Advanced Prostate Cancer, 2014

[103] S. R. Daigle, E. J. Olhava, C. A. Therkelsen et al., "Potent inhibition of DOT1L as treatment of MLL-fusion leukemia," Blood, vol. 122, no. 6, pp. 1017-1025, 2013.

[104] E. Olhava, R. Chesworth, K. Kuntz, V. Richon, R. Pollock, and S. Daigle, "Preparation of substituted purine and 7-deazapurine compounds as modulators of epigenetic enzymes," PCT Int.Appl.WO, 2011.

[105] A. Basavapathruni, E. J. Olhava, S. R. Daigle et al., "Nonclinical pharmacokinetics and metabolism of EPZ-5676, a novel DOT1L histone methyltransferase inhibitor," Biopharmaceutics and Drug Disposition, vol. 35, no. 4, pp. 237-252, 2014.

[106] W. Yu, E. J. Chory, A. K. Wernimont et al., "Catalytic site remodelling of the DOT1L methyltransferase by selective inhibitors," Nature Communications, vol. 3, article 1288, 2012.

[107] Y. Yao, P. Chen, J. Diao et al., "Selective inhibitors of histone methyltransferase DOT1L: design, synthesis, and crystallographic studies," Journal of the American Chemical Society, vol. 133, no. 42, pp. 16746-16749, 2011. 


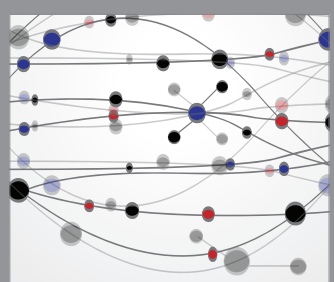

The Scientific World Journal
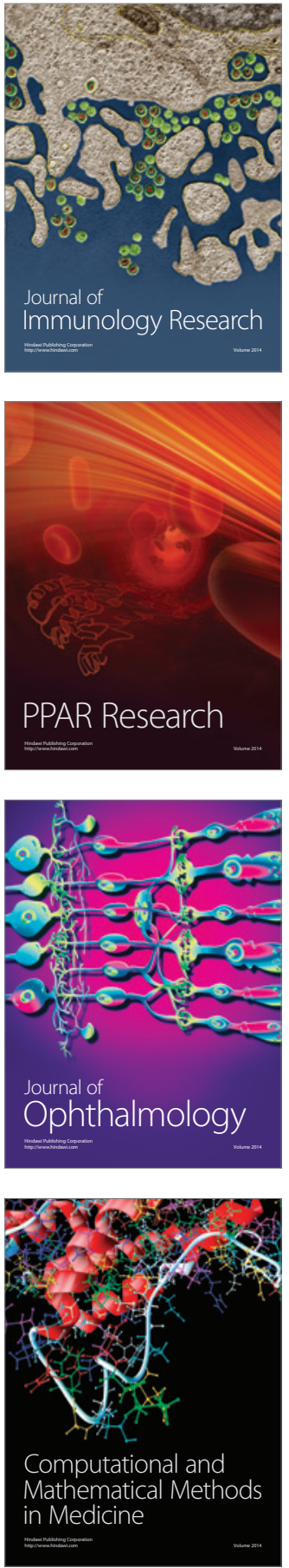

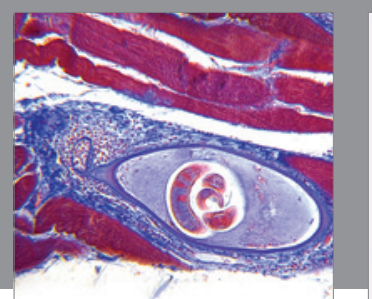

Gastroenterology Research and Practice

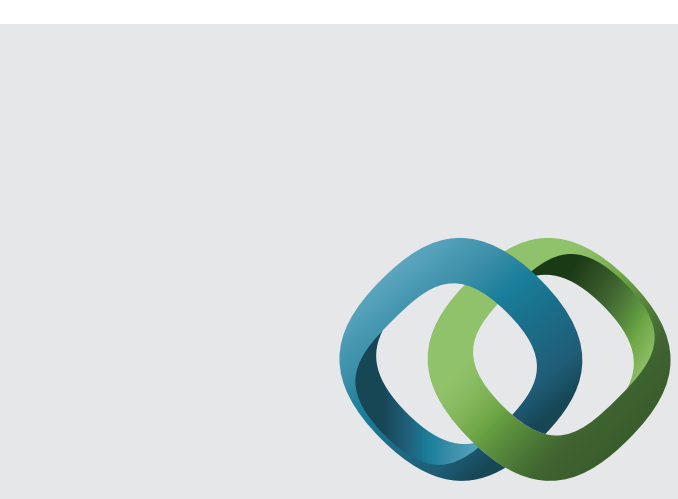

\section{Hindawi}

Submit your manuscripts at

http://www.hindawi.com
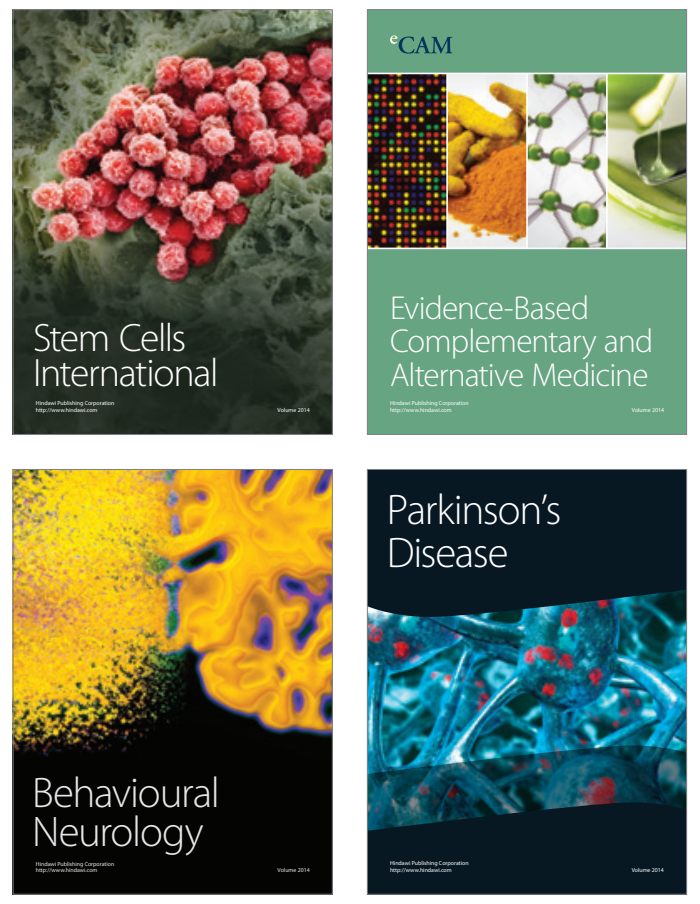
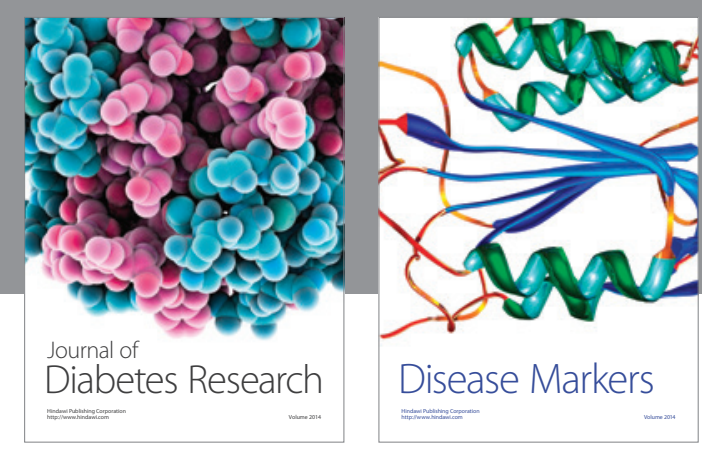

Disease Markers
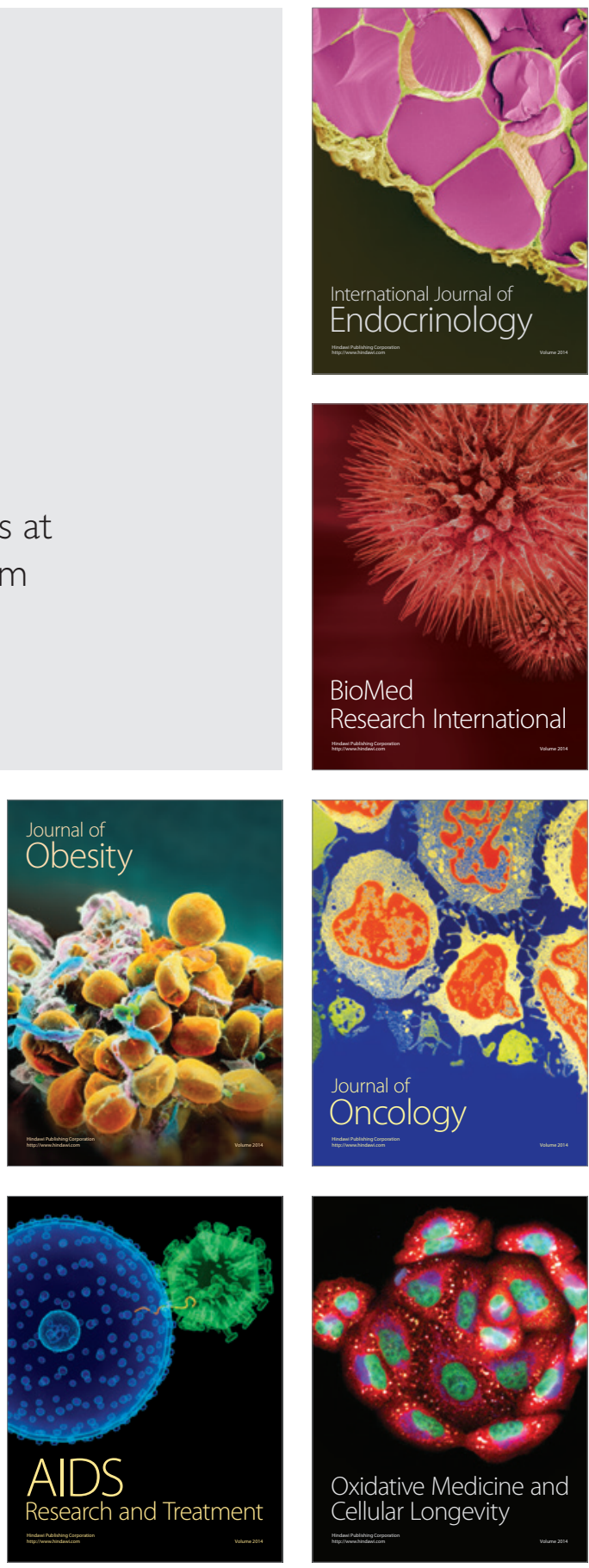